\title{
Speciation and Antibiotic Susceptibility Pattern of Shigella Isolates in a Rural Tertiary Care Hospital, Kolar
}

\author{
Parimala Subramani* (D), Bharathi Ramachandra, \\ Beena Pavangada Madappa and Arvind Natarajan \\ Department of Microbiology, Sri Devaraj URS Academy of Higher Education and Research (SDUAHER), \\ Tamaka, Kolar - 563 101, Karnataka, India.
}

\begin{abstract}
Shigellosis is the most important cause of bacillary dysentery. It's a major public health problem. It is endemic in many developing countries resulting in a significant amount of mortality and morbidity. The incidence is 90 million with 6,00,000 deaths world over. In India, there is an emergence of resistance to Shigella resulting in limitation of treatment. To study the prevalence of Shigella species and their antibiogram in stool samples obtained from patients treated for dysentery at Rural Tertiary Care Hospital. A retrospective analysis of the Shigella isolates obtained from 400 stool samples from patients with dysentery, treated at Rural Tertiary Care Hospital from 2015 -2019 was conducted. The isolates were identified by conventional methods and antibiotic susceptibility testing was performed by Kirbybauers disk diffusion method and analysed. The percentage of isolation of Shigella species at our centre was $\mathbf{5 \%}$ with Shigella flexneri being the predominant species isolated. A progressive pattern of resistance was observed. $100 \%$ strains of Shigella flexneri and Shigella sonnei were resistant to cotrimoxazole and nalidixic acid. $\mathbf{7 5 \%}$ of Shigella flexneri were resistant to fluoroquinolones and $\mathbf{2 5 \%}$ resistant to third generation cephalosporins. $100 \%$ isolates were sensitive to azithromycin. In view of rampant resistance observed among Shigella species, it is a need to test these isolates for antibiotic susceptibility and treat the patients based on the antibiogram.
\end{abstract}

Keywords: Shigellosis, dysentery, antibiotic resistance

*Correspondence: mjchand@gmail.com; +91 9740189773

(Received: August 21, 2021; accepted: November 18, 2021)

Citation: Subramani P, Ramachandra B, Madappa BP, Natarajan A. Speciation and Antibiotic Susceptibility Pattern of Shigella Isolates in a Rural Tertiary Care Hospital, Kolar. J Pure App/ Microbiol. 2022;16(1):276-280. doi: 10.22207/JPAM.16.1.16

(C) The Author(s) 2022. Open Access. This article is distributed under the terms of the Creative Commons Attribution 4.0 International License which permits unrestricted use, sharing, distribution, and reproduction in any medium, provided you give appropriate credit to the original author(s) and the source, provide a link to the Creative Commons license, and indicate if changes were made. 


\section{INTRODUCTION}

Shigellosis is a gastrointestinal infection caused by genus Shigella. ${ }^{1}$ One of the most common causes of morbidity and mortality among children in developing countries is acute gastroenteritis due to Shigella species. Shigella is a gram-negative bacilli that belongs to the family Enterobacteriaceae and is generically very similar to Escherichia coli. ${ }^{2}$ Genus Shigella is divided into Shigella dysenteriae, Shigella flexneri, Shigella boydii and Shigella sonnei. ${ }^{3}$ The mode of transmission is faeco-oral and the infective dose is as low as 10 -to 100 bacilli. The pathogen colonizes and disrupts the intestinal epithelium resulting in fever, bloody diarrhoea and tenesmus. ${ }^{4}$ One of the most common causes of morbidity and mortality among children in developing countries is acute gastroenteritis due to Shigella species. It predominantly affects children below 5 years, elderly and immunocompromised. It accounts for 1.1 million deaths in developing countries, of which $60 \%$ is constituted by children below 5 years of age. ${ }^{5}$ The mortality and morbidity due to Shigellosis is preventable by oral rehydration and appropriate antimicrobial agents. WHO reports of Multidrug-resistant (MDR) Shigella in India, and other developing countries has become a therapeutic challenge in the treatment of Shigellosis. ${ }^{1,6}$ So, this study is undertaken to know about the prevalence of Shigella species and their antibiogram in stool samples obtained from patients treated for dysentery at Rural Tertiary Care Hospital.

This study helps us understand the changing trend of antibiotic resistance among Shigella isolates in a rural background where antibiotic usage is comparatively low.

\section{Objectives}

To study the prevalence of Shigella species and their antibiogram in stool samples obtained from patients treated for dysentery.

\section{MATERIALS AND METHODS}

A retrospective analysis of the Shigella isolates from stool culture of patients with dysentery, treated at RL Jalappa Hospital from May 2015 - June 2019 was conducted. Ethical clearance was obtained from the institutional ethics committee.

\section{Inclusion criteria}

Shigella isolates from patients who presented with dysentery were included

\section{Exclusion criteria}

Incomplete patient data.

20 isolates of Shigella from patients who presented with dysentery were included in the study and were identified by conventional culture methods. The stool specimens were inoculated on Blood agar, Mac Conkey agar, Xylose lysine deoxycholate (XLD) agar directly. They were also inoculated into enrichment media, Selinite F broth and subcultured to MacConkey and XLD agar. Suspected colonies of Shigella was identified by standard biochemical tests and confirmed by specific antiserum (Denka -Seiken, Japan). Antibiotic susceptibility testing was done by Kirby Bauer disk diffusion technique as per CLSI (Clinical and Laboratory standard institute) guidelines using the following antibiotics, ampicillin (Amp)10 $\mu$ g, cotrimoxazole (Cot) $25 \mu \mathrm{g}$, nalidixic acid ( $\mathrm{Na}) 30 \mu \mathrm{g}$, ciprofloxacin (Cip) $5 \mu \mathrm{g}$, and ceftriaxone (Ctr) 30 $\mu \mathrm{g}$ (Hi Media Laboratories, India). An additional drug azithromycin (Az) 15 $\mathrm{gg}$ was tested as an investigational drug to find the usefulness of the drug as second line drug in case of pan drug- resistance. These isolates were further confirmed by NICED (National Institute of Cholera and Enteric Disease) Kolkotta. Further, their antibiotic susceptibility pattern was analysed as per CLSI (Clinical and Laboratory standard institute) guidelines. The data was retrieved from the Medical records department.

Statistical Analysis

A descriptive analysis of the data was done.

\section{RESULTS}

A total number of 400 stool samples were cultured between May 2015-June 2019. $20(5 \%)$ isolates of Shigella species were obtained from patients with dysentery, treated at Rural Tertiary Care Hospital. Out of the 20 isolates, the predominant serotype was Shigella flexneri, 16 (80\%) followed by Shigella sonnei, 4 (20\%). A gradual increase in the number of isolates from 2015 to 2019 was observed as shown in (Table 1$)$. The majority of the isolates $18(80 \%)$ 
Table 1. Distribution of shigella isolates during 5 years period

\begin{tabular}{|c|c|c|c|}
\hline$N=20$ & & Shigella flexneri (6) 80\% & Shigella sonnei (4) $20 \%$ \\
\hline 2015 & 2 & Shigella flexneri 4A & \\
\hline & & Shigella flexneri $2 \mathrm{~A}$ & NIL \\
\hline 2016 & 2 & Shigella flexneri 1B & Shigella sonnei Phase $2(3)$ \\
\hline & & Shigella flexneri $2 \mathrm{~A}$ & \\
\hline 2017 & 3 & Shigella flexneri $2 \mathrm{~A}$ & NIL \\
\hline & & Shigella flexneri $3 \mathrm{~A}$ & \\
\hline 2018 & 2 & Shigella flexneri & NIL \\
\hline & & Shigella flexneri & \\
\hline 2019 & 7 & Shigella flexneri $2 \mathrm{~A}$ & Shigella sonnei Phase 2(1) \\
\hline & & Shigella flexneri & \\
\hline
\end{tabular}

Note: A and B denotes the serotypes of Shigella.

Table 2. Resistance pattern in shigella isolates $(\mathrm{N}=20)$

\begin{tabular}{lccc}
\hline Antibiotic & Shigella flexneri (16) & Shigella sonnei (4) & Total (20) \\
\hline AMPICILLIN & $13(81.25 \%)$ & NIL & 13 \\
COTRIMOXAZOLE & $16(100 \%)$ & $4(100 \%)$ & 20 \\
NALIDIXIC ACID & $16(100 \%)$ & $4(100 \%)$ & 20 \\
CIPROFLOXACIN & $12(75 \%)$ & NIL & 12 \\
CEFTRIAXONE & $4(25 \%)$ & NIL & 4 \\
AZITHROMYCIN & $16(100 \%)$ & NIL & 16 \\
\hline
\end{tabular}

Note: The figure shown in the bracket is resistant.

out of 20 isolates were from the adult population. All the 20 isolates showed $100 \%$ resistance to cotrimoxazole and nalidixic acid as shown in (Table 2). 4 (25\%) isolates of Shigella flexneri showed resistance to ceftriaxone, 2 of the 4 were ESBL producers. All the $20(100 \%)$ isolates were sensitive to azithromycin.

\section{DISCUSSION}

Global estimation of shigellosis is $\mathbf{1 6 4 . 7}$ million annually. ${ }^{7}$ One of the major public health problems in developing countries is shigellosis. It is transmitted through contaminated food and water and direct contact with infected individuals. In the present study, the rate of isolation was $5 \%$ which is higher than the rate quoted by Taneja et al. ${ }^{8}$ The overall percentage of isolation of Shigella from different parts of our country ranges from 3 to $6 \%$. It has been shown by many studies that shigellosis is prevalent among children less than 5 years. ${ }^{9}$ Contrary to the above observation made, the majority of the isolates 18 (80\%) out of 20 were from the adult population in the present study.

The most common serotype isolated in our study is Shigella flexneri followed by Shigella sonnei. Madhavan et al. has reported Shigella sonnei as the predominant serotype in Kerala. ${ }^{8}$ Shigella sonne ${ }^{9}$ was found to be the predominant species in Thailand, Vietnam and Srilanka attributed to improved sanitary conditions. From various studies across the world, it is found that there is a shift from Shigella flexneri to Shigella sonnei in countries with improved sanitation. ${ }^{9}$

The trend of antibiotic resistance was progressive in our study. An alarming increase in the resistance pattern of Shigella flexneri was observed. 13 (81\%) of the Shigella flexneri strains were MDR strains resistant to more than three classes of antibiotics. 12 (75\%) of the strains were resistant to ciprofloxacin. Fluoroquinolones were recommended by the WHO as drug of choice in 1990 but the emergence of resistance 
to fluoroquinolone has limited its usage and has mandated the use of ceftriaxone. The emergence of fluoroquinolone resistance is probably due to the over use of these drugs for diarrhoea and urinary tract infections. 5.9\% of fluoroquinolone resistance was observed in 2004 which increased to $48.5 \%$ in $2007 .{ }^{9}$ Gururaj et al. proved that there was a uniform mutation in Gyr A among the fluoroquine resistant strains. ${ }^{10}$ Presently some of the strains have developed resistance to third-generation cephalosporins. Madhavan et al. ${ }^{11}$ showed that $17 \%$ of Shigella isolates in his study were resistant to third-generation cephalosporin as compared to $25 \%$ in our study. Mandal J et al. showed $3 \%$ of cephalosporin resistance which was lesser in comparison to our study. ESBL production was observed in $2(12.5 \%)$ isolates of Shigella flexneri as compared to $3 \%$ in the study conducted by Mandal J et al. ${ }^{12}$ The greatest danger faced with MDR strains are the limited therapeutic options available to the treating physician. ${ }^{13}$ In a rural setup, the cost of procuring higher antibiotics results in delayed recovery and prolonged hospital stay, on the isolation of an MDR strain.

As CLSI recommends azithromycin to be tested as an investigational drug, azithromycin sensitivity was performed on all the isolates obtained in the year 2019 and $100 \%$ sensitivity was observed. Although there is a smaller sample size this work has gained success to fulfil the purpose of this work to achieve better treatment

\section{Limitation of the Study}

The sample size is the limitation of the study. Extrapolation of the results could have been better with a larger sample size.

\section{CONCLUSION}

Shigella flexneri has become increasingly resistant to antimicrobials. WHO in 2005 recommended ciprofloxacin as the first line treatment for shigellosis in children and ceftriaxone as a second line drug for children. Azithromycin as a second line drug for adults. Shigellosis should be treated with antibiotics as it shortens the duration of clinical symptoms and decreases fecal excretion of the organism and minimises transmission. Provision for improved sanitation and clean drinking water is needed to prevent shigellosis. There is a impending need for updation of the guidelines for treating shigellosis in the developing countries

This work would ameliorate the treatment for dysentery, it exhibits a significance in the treatment among rural areas where in many cases are not diagnosed and treated effectively. Instead of the limitation due to the smaller sample size this work is very fruitful for the study of the prevalence of Shigella species and antibiogram which is very important for the proper treatment of patients with dysentery.

\section{ACKNOWLEDGMENTS}

None.

\section{CONFLICT OF INTEREST}

The authors declare that there is no conflict of interest.

\section{AUTHORS' CONTRIBUTION}

PS designed the study and implemented the concepts. PS and BR did the literature search, Data acquisition, Data analysis and wrote the manuscript. BPM, AN and PS reviewed and edited the manuscript.

\section{FUNDING}

None.

\section{DATA AVAILABILITY}

All datasets generated or analyzed during this study are included in manuscript.

\section{ETHICS STATEMENT}

This study was approved by the Institutional Ethics Committee, Sri Devaraj URS Medical College, Tamaka, Karnataka, India.

\section{REFERENCES}

1. Prabhurajeshwar, Oli KA, Ashajyothi C, Chandrakanth $K$. Prevalence and antibiotic susceptibility of fluoroquinolone resistant Shigella species isolated from infants stool in Gulbarga district, Karnataka, India. Asian Pac J Trop Dis. 2015;5(Suppl 1):S116-S120. doi: 10.1016/S2222-1808(15)60871-4

2. Schroeder GN, Hilbi H. Molecular pathogenesis of Shigella species: Controlling host cell signalling, invasion and death by type III secretion. Clin Microbiol Rev. 2008;21(1):134-156. doi: 10.1128/CMR.00032-07

3. Dupont HL. Shigella species (Bacillary Dysentry) In: Mandell GL, Bennett JE, Dolin R, Editors. Principles and practice of infectious diseases. Churchil Livingstone 
Elseiver; Philadelphia; 2010:2905-2910.

4. Jain PA, Kulkarni RD, Dutta S, et al. Prevalence and antimicrobial profile of Shigella isolates in a tertary care hospital of North Karnataka; A 12 year study. Indian J Med Microbiol. 2020;38(1):101-118. doi: 10.4103/ijmm.IJMM_20_107

5. Shakya G, Acharya J, Adhikari S, Rijal N. Shigellosis in Nepal: 13 years review of nation wide surveillance. J Health Popul Nutr. 2016;35(1):36. doi: 10.1186/ s41043-016-0073-x

6. WHO: Antimicrobial resistance: global report on surveillance. 2014. WHO Press, World Health Organization 20 Avenue Appia, 1211 Geneva 27, Switzerland.

7. Puzari M, Sharma M, Chetia P. Emergence of antibiotic resistant Shigella species: A matter of concern. J Infect Public Health. 2018;11(4):451-454. doi: 10.1016/j. jiph.2017.09.025

8. Taneja N, Lyndoh V, Vermani A, et al. The re-emergence of multidrug resistant strains of Shigella dysenteriae with an added resistance to ciprofloxacin in north
India and their plasmid profile. Indian J Med Res. 2005;122(4):348-354.

9. Jesudason MV. Shigella isolation in Vellore, South India (1997-2001). Indian J Med Res. 2002;115:11-13.

10. Pazhani GP, Niyogi SK, Singh AK, et al. Molecular characterization of multidrug resistant Shigella species isolated from epidemic and endemic cases of Shigellosis in India. J Med Microbiol. 2008;57(Pt 7): 856-863. doi: 10.1099/jmm.0.2008/000521-0

11. Madhavan A, Balakrishna S, Vasudevapanicker J. Antibiotic susceptibility pattern of Shigella isolates in a tertiary health care centre. J lab Physicians. 2018;10(2):140-144. doi: 10.4103/JLP.JLP_93_17

12. Mandal J, Ganesh V, Emelda J, Mahadevan S, Parija SC. The recent trends of Shigellosis : A Jipmer perspective. J Clin Diagn Res. 2012;6(9):1474-1477. doi: 10.7860/ JCDR/2012/4157.2536

13. Gajdacs $M$. The concept of an ideal antibiotic : Implication of drug design. Molecules. 2019;24(5):892. doi: $10.3390 /$ molecules 24050892 